\title{
Multiple herbicide resistance evolution: The case of Eleusine indica in Brazil
}

José G. Vázquez-García ${ }^{1}$, Ricardo Alcántara de la Cruz $^{2 *}$ Antonia M. Rojano-Delgado ${ }^{1}$, Candelario Palma-Bautista ${ }^{1}$, João M. de Portugal Vasconcelos $^{3,4}$ and Rafael De Prado ${ }^{1}$

${ }^{1}$ Department of Agricultural Chemistry and Edaphology, University of Cordoba, 14071-Cordoba, Spain.

${ }^{2}$ Departamento de Química, Universidade Federal de São Carlos, São Carlos 13565-905, Brasil.

${ }^{3}$ Biosciences Department, Polytechnic Institute of Beja, 7800-295 Beja, Portugal.

${ }^{4}$ VALORIZA-Research Centre for Endogenous Resource Valorization, Polytechnic Institute of Portalegre, 7300-555 Portalegre, Portugal

*Corresponding author: ricardo.cruz@ufscar.br (R.A.C.).

Table S1. ${ }^{14} \mathrm{C}$-herbicide used for absorption and translocation studies.

\begin{tabular}{|c|c|c|c|c|}
\hline Herbicide & Provider & Specific activity & Rinsing solution & Reference \\
\hline${ }^{14} \mathrm{C}$-diclofop-methyl & INRA Dijon, France & & Water-acetone $(1: 1 \mathrm{v} / \mathrm{v})$ & 16 \\
\hline${ }^{14} \mathrm{C}$-imazamox ${ }^{\mathrm{a}}$ & BASF, Germany & & Water-methanol $(1: 9 \mathrm{v} / \mathrm{v})$ & 17 \\
\hline${ }^{14} \mathrm{C}$-paraquat & American Radiolabeled Chemical, USA & $6.2789 \mathrm{MBq} / \mathrm{mg}$ & Water-acetone $(1: 1 \mathrm{v} / \mathrm{v})$ & 18 \\
\hline${ }^{14} \mathrm{C}$-glyphosate & American Radiolabeled Chemical, USA & $273.8 \mathrm{MBq} \mathrm{mmol}^{-1}$ & Water-acetone $(1: 1 \mathrm{v} / \mathrm{v})$ & 19 \\
\hline
\end{tabular}

a plus $1.25 \mathrm{~L} \mathrm{ha}^{-1}$ of Dash 
Table S2. Herbicide metabolism methodologies, its references and technical characteristics.

\begin{tabular}{|c|c|c|c|c|c|c|}
\hline Herbicide & Extraction & $\begin{array}{l}\text { Separation } \\
\text { techniques }\end{array}$ & Mobile phases & Detector & Metabolite studies & Reference \\
\hline $\begin{array}{l}{ }^{14} \mathrm{C} \text {-diclofop- } \\
\text { methyl }\end{array}$ & $\begin{array}{l}\text { Pulverized } \mathrm{N}_{2}+4 \mathrm{~mL} \text { of } 80 \% \\
\text { methanol }\end{array}$ & TLC & $\begin{array}{l}\text { toluene/ethanol/acetic } \\
\text { acid }(150 / 7 / 7, \mathrm{v} / \mathrm{v} / \mathrm{v})\end{array}$ & $\begin{array}{l}\text { Radioactivity } \\
\text { Detector }\end{array}$ & $\begin{array}{l}\text { D-acid, } \\
\text { D-hydroxy, D-cojugated }\end{array}$ & 16 \\
\hline Imazamox & $\begin{array}{l}\text { Pulverized } \mathrm{N}_{2} \text { assisted by } \\
\text { ultrasounds }(70 \mathrm{~W} \text { and } 0.7 \mathrm{~s} / \mathrm{s} \\
\text { duty cycle })+10 \mathrm{~mL} \\
\text { methanol-water }(9: 1, \mathrm{v} / \mathrm{v})\end{array}$ & $\mathrm{LC}$ & $\begin{array}{l}1 \%(\mathrm{v} / \mathrm{v}) \text { acetic acid in } \\
\text { water as mobile phase } \\
\text { A and pure methanol as } \\
\text { mobile phase B }\end{array}$ & DAD & $\begin{array}{l}\text { Hydroxy metabolite, } \\
\text { Glucose conjugated } \\
\text { metabolite }\end{array}$ & 20 \\
\hline Glyphosate & $\begin{array}{l}\text { Pulverized } \mathrm{N}_{2} \text { assisted by } \\
\text { short cycles of ultrasounds ( } 5 \\
\text { min) and magnetic stirring } \\
(10 \mathrm{~min})+8 \mathrm{~mL} \text { water- } \\
\text { acetone }(1: 1, \mathrm{v} / \mathrm{v})\end{array}$ & $\mathrm{CE}$ & $\begin{array}{l}\text { BGE with pH } 7.5=10 \% \\
\text { ACN, } 7.5 \quad \mathrm{mM} \\
\text { phthalate, } 0.75 \mathrm{mM} \\
\text { hexadecyltrimethylamm } \\
\text { onium bromide }\end{array}$ & $\begin{array}{l}\text { DAD with } \\
\text { indirect } \\
\text { detection }\end{array}$ & $\begin{array}{l}\text { AMPA, Glyoxylate, } \\
\text { Sarcosine, Formaldehyde }\end{array}$ & 21 \\
\hline
\end{tabular}

TLC - thin-layer chromatography; LC - liquid chromatography; CE - capillary electrophoresis; DAD - diode array detector; and AMPA aminomethylphosphonic acid. 
Table S3. Enzymatic activities methodologies, doses, substrates, main reactive, technical characteristics and references.

\begin{tabular}{|c|c|c|c|c|c|c|}
\hline Herbicide & Enzyme & Herbicide doses $(\mu \mathrm{M})$ & Substrates and some reactives & Measured substance & Detector & Reference \\
\hline $\begin{array}{l}\text { *Haloxyfop-p- } \\
\text { methyl } \\
\text { *Diclofop-methyl } \\
\text { Sethoxydim } \\
\text { Tralkoxydim } \\
\text { Pinoxadem }\end{array}$ & ACCase & $\begin{array}{l}0,0.01,0.1,1,10,100, \\
1000,5000\end{array}$ & $\begin{array}{l}\text { Acetyl-CoA, } \\
\mathrm{NaH}\left({ }^{14} \mathrm{C}\right) \mathrm{O}_{3}\end{array}$ & ${ }^{14} \mathrm{C}$-Malonyl-CoA & LSS & 17 \\
\hline Imazamox & ALS & $\begin{array}{l}0,0.01,0.1,1,10,100, \\
1000,5000\end{array}$ & $\begin{array}{l}\text { Pyruvate, FAD, creatine, } \boldsymbol{\alpha}- \\
\text { naphthol }\end{array}$ & $\begin{array}{l}\text { Reaction of acetoine } \\
\text { and } \boldsymbol{\alpha} \text {-naphthol }\end{array}$ & $\begin{array}{l}\text { Spectrophotometer } \\
\lambda 520 \mathrm{~nm}\end{array}$ & 18 \\
\hline Glyphosate & EPSPS & $\begin{array}{l}0,0.01,0.1,1,10,100, \\
10005000\end{array}$ & $\begin{array}{l}\text { Shikimate-3-phosphate }+ \\
\text { PEP, MESG, purine- } \\
\text { nucloside phosphorylase }\end{array}$ & $\mathrm{Pi}$ & $\begin{array}{l}\text { Spectrophotometer } \\
\lambda 360 \mathrm{~nm}\end{array}$ & 23 \\
\hline
\end{tabular}

* in acid form. ACCase - acetyl-CoA carboxylase; ALS - acetolactate synthase; EPSPS - 5-enolpyruvylshikimate-3-phosphate synthase; ATP adenosine triphosphate; FAD - flavin adenine dinucleotide; PEP - phosphoenolpyruvate; MESG - 7- methyl-purine riboside; and LSS - liquid scintillation spectrometry. 


\section{REFERENCES}

17. Golmohammadzadeh, S.; Rojano-Delgado, A.M.; Vázquez-García, J.G.; et al. Cross-resistance mechanisms to ACCase-inhibiting herbicides in short-spike canarygrass (Phalaris brachystachys). Plant Physiol. Biochem. 2020, 151, 681-688.

18. Rojano-Delgado, A.M.; Portugal, J.M.; Palma-Bautista, C.; et al. Target site as the main mechanism of resistance to imazamox in a Euphorbia heterophylla biotype. Sci. Rep. 2019. 9, 15423.

19. Tahmasebi, B.K.; Alcántara-de la Cruz R.; Alcántara, E.; et al. Multiple resistance evolution in bipyridylium-resistant Epilobium ciliatum after recurrent selection. Front. Plant Sci. 2018, 9, 695.

20. Alcántara-de la Cruz, R.; Fernández-Moreno, P.T.; Ozuna, C. V.; et al. Target and non-target site mechanisms developed by glyphosateresistant hairy beggarticks (Bidens pilosa L.). Front. Plant Sci. 2016, 7, 1492.

21. Rojano-Delgado, A.M.; Priego-Capote, F.; De Prado, R.; Luque de Castro, M.D. Ultrasound-assisted extraction with LC-TOF/MS identification and LC-UV determination of imazamox and its metabolites in leaves of wheat plants. Phytochem. Anal. 2014, $25: 357-363$.

22. Rojano-Delgado, A.M.; Ruíz-Jiménez, J.; Luque de Castro, M.D.; De Prado, R. Determination of glyphosate and its metabolites in plant material by reversed-polarity CE with indirect absorptiometric detection. Electrophoresis. 2010, 31, 1423-1430.

23. Dayan, F.E.; Owens, D.K.; Corniani, N.; et al. Biochemical markers and enzyme assays for herbicide mode of action and resistance studies. Weed Sci. 2015, 63, 23-63. 